10

TRANS · núm. I $7 \cdot 2013$

DOSSIER $\cdot 71-84$
La compensación es una estrategia empleada con frecuencia en traducción audiovisual con el fin de superar las restricciones que caracterizan a los textos audiovisuales (Chaume, 2008: 82). En el caso del doblaje, aparte de las restricciones motivadas por el ajuste de la versión doblada, el traductor debe tener en cuenta que el texto que llega a sus manos ha sido escrito para ser interpretado como si no hubiese sido escrito, es decir, como si fuese espontáneo. Además, el traductor deberá considerar que el registro del doblaje cuenta con características propias y que determinadas marcas de oralidad frecuentes en los textos audiovisuales originales no son apropiadas en la traducción para el doblaje (Baños Piñero y Chaume, 2009). Dada la imposibilidad de utilizar en la versión doblada determinados recursos lingüisticos que dotan de espontaneidad al texto audiovisual, la compensación se torna una estrategia particularmente útil para conseguir unos diálogos creibles. El objetivo de este artículo es reflexionar sobre la idoneidad de esta estrategia para recrear la conversación espontánea en la versión doblada. Para ello, se ofrecerán ejemplos del doblaje de la comedia de situación Friends al español, en los que el traductor recurre a la compensación mediante la introducción de rasgos propios de la conversación coloquial espontánea que están ausentes en el original.

PALABRAS CLAVE: compensación, estrategia de traducción, doblaje, oralidad prefabricada, dubbese, comedia de situación.

\title{
La compensación como estrategia para recrear la conversación espontánea en el doblaje de comedias de situación
}

\section{Compensation: a translation strategy to mirror spontaneous-sounding conversation when dubbing sitcoms}

Compensation is a translation strategy used frequently in audiovisual translation in order to overcome the constraints which characterize audiovisual texts (Chaume, 2008: 82). In dubbing, in addition to considering synchronization constraints, translators should take into account that the text they receive has been written to be interpreted as if it had not been written, that is, as if it was spontaneous. In addition, translators should bear in mind the specificities of the register of dubbing, and that some orality markers which are common in domestic productions, are not appropriate in dubbed productions (Baños Piñero and Chaume, 2009). Due to the impossibility of using specific linguistic features which make audiovisual dialogue sound spontaneous, compensation turns out to be a very useful strategy to achieve credible dialogues in dubbed productions. The aim of this article is to reflect on the appropriateness of this strategy to mirror naturally-occurring conversation in dubbing. This will be illustrated with examples from episodes of the TV series Friends dubbed into Spanish. Through these examples, it will be shown how compensation is implemented through the addition of features which are typical of colloquial conversation in Spanish and which are absent in the original version.

KEY WORDS: compensation, translation strategy, dubbing, prefabricated orality, dubbese, situation comedy. 


\section{INTRODUCCIÓN}

Desde el surgimiento de los medios audiovisuales y tras su consolidación como medio de comunicación de masas, se han dedicado grandes esfuerzos a describir y descubrir los entresijos del discurso audiovisual, considerado como un lenguaje propio y específico, caracterizado por la complejidad de los códigos de significación que lo conforman. Casetti y Di Chio (r99r) definen el lenguaje cinematográfico como un lenguaje abigarrado que combina varios tipos de significantes (imágenes, música, ruidos, palabras y textos) y de signos (índices, iconos y símbolos). De igual modo, basándose en los códigos de significación del lenguaje fílmico, Chaume (2004) establece un marco de análisis de los textos audiovisuales con fines traductológicos, en el que distingue los códigos de significación transmitidos a través del canal acústico y aquellos transmitidos mediante el canal visual.

De entre estos códigos de significación, el código lingüístico es el de mayor relevancia en traducción. Sin ir más lejos, este código es el responsable de que los textos audiovisuales sean susceptibles de ser traducidos. Asimismo, el código lingüístico (transmitido mediante el canal acústico) de los textos audiovisuales se caracteriza no solo por su peculiar modo de transmisión, sino también por el modo de producción y recepción del discurso (Salvador, 1989: 15). En este sentido, el texto audiovisual suele partir de un texto escrito con antelación que ha de ser interpretado por los actores como si no hubiese sido escrito (Gregory y Carroll, I978: 47). Por este motivo, podemos hablar de un discurso oral elaborado o prefabricado, que comparte numerosas características con el discurso oral espontáneo (al que pretende imitar), pero que cuenta con otras tantas características propias de la escritura.
En el caso del doblaje, las características propias del discurso audiovisual en la lengua origen se transmiten mediante el lenguaje oral en lengua meta, por lo que podría parecer que la imitación del discurso oral espontáneo no debería presentar gran dificultad. No obstante, al enfrentarse a este reto, el traductor que también se encargue de la adaptación de diálogos tendrá que tener en cuenta no solo las restricciones propias del doblaje relacionadas con la sincronía, sino también aquellas limitaciones fruto de la normalización lingüística y estilística que, según Ávila (r997: 25-26), recomiendan los estudios de doblaje para satisfacer las necesidades del espectador. Para ello, será necesario desarrollar una serie de estrategias que le permitan trasladar las distintas intervenciones de los personajes en pantalla de tal manera que, al escucharlas o leerlas, suenen naturales y espontáneas, y puedan ser identificadas y comprendidas con facilidad por los espectadores. Entre otras, la compensación es una de las estrategias que puede emplear el traductor audiovisual para superar las restricciones que caracterizan a los textos audiovisuales (Chaume, 2008: 82). De hecho, consideramos que se trata de una estrategia especialmente eficaz a la hora de establecer el equilibrio entre lo oral y lo escrito en el diálogo doblado, ya no solo debido a la especificidad del doblaje, sino también a la particular naturaleza de su oralidad.

El objetivo de este artículo es reflexionar sobre la eficacia de la estrategia de compensación a la hora de suplir la ausencia de determinados rasgos lingüísticos que dotan de espontaneidad al texto audiovisual original, con el fin de recrear la conversación espontánea en la versión doblada. Para ello, se ofrecerán ejemplos del doblaje de la comedia de situación Friends al español en los que 
el traductor ${ }^{1}$ recurre a rasgos propios de la conversación coloquial espontánea que están ausentes en el original. Sin embargo, antes de reflexionar sobre el carácter prefabricado del discurso del doblaje y de presentar ejemplos que ilustren cómo se ha utilizado la compensación para crear un discurso pretendidamente espontáneo, será necesario que definamos qué entendemos exactamente por compensación.

\section{LA COMPENSACIÓN EN LOS ESTUDIOS DE TRADUCCIÓN}

Son varios los autores que incluyen la compensación en las clasificaciones de las estrategias, procedimientos o técnicas de traducción (en función de la terminología empleada en cada caso $^{2}$ ). Sin embargo, según apunta Harvey (1995: 65), se trata de un término que no siempre se ha definido de forma clara y sistemática, y que parece estar sujeto a distintas interpretaciones. Este autor define la compensación como «a

I Aunque nos refiramos en general al traductor (y en particular al traductor que se encarga del ajuste de los diálogos), es preciso tener en cuenta que son varios los profesionales que intervienen en el proceso del doblaje y que es muy complicado determinar con certeza si el traductor es el responsable de la selección de determinados recursos lingüísticos presentes en la versión doblada. De hecho, tal y como apunta Richart en este dossier, los cambios que se realizan durante el ajuste y la grabación de la versión doblada son considerables, y es probable que sean otros los agentes responsables de la introducción de algunas marcas de oralidad en la versión española. Por tanto, en este artículo se deberá entender que el término traductor engloba a todos los profesionales que participan en el proceso del doblaje y que contribuyen a la creación de los diálogos en la versión doblada.

2 El propósito de este artículo no es ilustrar las distintas opiniones respecto a si debemos considerar la compensación un procedimiento, una técnica o una estrategia. En nuestro caso, coincidimos con Chaume (2008: 73) en que se trata de una estrategia de traducción, que se puede materializar mediante diversas técnicas en el texto meta, y remitimos al lector a dicho trabajo para profundizar sobre esta cuestión. technique for making up for the loss of a source text effect by recreating a similar effect in the target text through means that are specific to the target language and/or to the target text» (Harvey, I995: 66).

Al igual que Harvey, la mayor parte de los teóricos considera que esta estrategia puede materializarse de muy diversas formas en el texto meta y que responde a la necesidad de compensar la pérdida o ausencia de un efecto concreto en el texto traducido - véanse, por ejemplo, Hatim y Mason (1997: 179) o Baker (201r: 86). Newmark (1988: 90), por ejemplo, defiende que la compensación tiene lugar «when loss of meaning, sound-effect, metaphor or pragmatic effect in one part of a sentence is compensated in another part, or in a contiguous sentence». Aunque similar, la definición que propone Hurtado (200r: 634) no hace referencia al lugar específico en el que se aplica la estrategia compensatoria, definida en este caso como una técnica de traducción que "consiste en introducir en otro lugar del texto un elemento de información o un efecto estilístico que no ha podido reflejarse en el mismo sitio en que está situado en el texto original». Dada la aparente falta de acuerdo a este respecto, Harvey (I995: 82-83) propone distinguir entre compensación «paralela», «contigua» o «desplazada», en función de la posición del elemento compensatorio con respecto al elemento del original que no se pudo reproducir en su momento. En el primer caso, la compensación tendría lugar exactamente en el mismo lugar en el texto origen y en el texto meta, mientras que el segundo tipo de compensación se situaría en el texto traducido a poca distancia de donde se encontraba originalmente el elemento del texto origen, que se «pierde» u omite en la traducción. En la compensación desplazada, el elemento compensatorio se introduce en otro lugar del texto 
meta y no en las inmediaciones del elemento del original que está ausente en la traducción.

Sin embargo, para Chaume (2008: 78), solo 74 tiene sentido hablar de compensación en este último caso, es decir, si la entendemos como «una estrategia que conduce al uso de una técnica de traducción en un lugar del texto meta en donde no se presentaba ninguna restricción ni problema de traducción en el segmento origen reemplazado». En este caso, no obstante, ¿cómo sabemos qué elemento del original está siendo reemplazado o compensado? El propio Harvey (I995: 84) advierte de la dificultad que entraña el análisis de la compensación desplazada, ya que resulta complicado (si no imposible) identificar el elemento del original reemplazado en cada caso y garantizar que el propósito del traductor era precisamente el de realizar tal sustitución. Con el fin de evitar juicios subjetivos, Harvey propone un último tipo de compensación, que denomina «generalized compensation» y que define del siguiente modo:

This occurs where the target text includes stylistic features that help to naturalize the text for the target reader and that aim to achieve a comparable number and quality of effects, without these being tied to any specific instances of source text loss (Harvey, I995: 84).

En este artículo nos referiremos a este tipo de compensación: hablaremos de una estrategia de compensación generalizada al considerar que, dada la naturaleza de nuestro estudio, solo podemos realizar juicios hipotéticos con respecto al origen y al motivo concreto de la introducción del elemento compensatorio analizado en el texto meta. Asimismo, coincidimos con Chaume (2008: 78) al considerar que, para poder hablar de compensación, es necesario que haya desplazamiento. Al adoptar este punto de vista en el análisis, tomamos como punto de partida el polo meta y consideramos el texto audiovisual en conjunto como unidad de traducción. En este caso, según afirma Chesterman (I997: II5), «if [...] the translator chooses to do something (add, omit, change etc.) at one point in the text, this action in itself may be sufficient justification for a compensatory strategy at some other point in the text». El propósito de la estrategia compensatoria consistirá, por lo tanto, en conseguir que el texto audiovisual traducido produzca un efecto similar o «equivalente» al original en los espectadores del texto meta (en nuestro caso, que los diálogos resulten creíbles y verosímiles), si bien la consecución de tal equivalencia es difícil de juzgar de forma sistemática (Harvey, r998: 39).

\section{LA COMPENSACIÓN EN TRADUCCIÓN AUDIOVISUAL}

En el ámbito de la traducción audiovisual, la compensación ha sido abordada por varios autores $^{3}$ probablemente debido a que se trata de una estrategia empleada con frecuencia en sus distintas modalidades (doblaje, subtitulado, voces superpuestas, etc.). En comparación con otras modalidades, puede que el traductor para el doblaje goce de mayor libertad a la hora de aplicar esta estrategia. En el caso del subtitulado, por ejemplo, a pesar de ser una estrategia habitual, «[...] it may not always be practicable due to the oral-visual cohabitation of the source and target languages: the subtitles should not deviate too much from the soundtrack if the readers/viewers can be expected to understand

3 Entre otros, cabe destacar el trabajo de Chaume (2008), que proporciona una definición del concepto de compensación desde el punto de vista de la traducción audiovisual, así como ejemplos que muestran la aplicación de esta estrategia. 
at least part of what they hear» (Díaz Cintas y Remael, 2007: 206).

La compensación implica un esfuerzo creativo considerable y, si bien se trata de una estrategia aplicable ante la presencia de problemas y restricciones de distinto tipo, el traductor debe discernir si tal esfuerzo merece la pena en cada caso (Newmark, I99r: 144). Se puede argüir, por tanto, que al traductor audiovisual le resultará más «rentable» aplicar esta estrategia al enfrentarse a determinados retos. Con frecuencia se menciona la eficacia de la compensación a la hora de afrontar la traducción del humor, de frases idiomáticas y de juegos de palabras. En el ámbito de la traducción audiovisual, por ejemplo, Chiaro (2005: 136) menciona esta estrategia para traducir «verbally expressed humour». Martínez Sierra (2008) va más allá del componente verbal e ilustra el uso de esta estrategia en el doblaje al español de Los Simpson, haciendo hincapié en la relevancia del código paralingüístico y de los códigos transmitidos mediante el canal visual. Sin embargo, según advierte Zabalbeascoa (200I), en algunos casos puede que la compensación no sea apropiada para la consecución del efecto cómico en la versión doblada. Este es el caso de las telecomedias con «risa enlatada», sobre todo si las risas de fondo no vienen grabadas en la pista de diálogos:

[...] si el traductor «se rinde» en algún chiste concreto, es decir, es plenamente consciente de que su versión no consigue el efecto humorístico donde aparece en el original, dejar las risas grabadas producirá una incoherencia que muy probablemente despistará, desorientará o molestará a la audiencia. [...] Si el traductor compensa el no haber podido lograr el efecto deseado en un lugar concreto introduciéndolo en otro, resulta que si el efecto es la risa en los espectadores y esto se ve favorecido por las risas grabadas, el problema será doble si tenemos risas grabadas donde no hay humor $\mathrm{y}$ no las tenemos ( $\mathrm{y}$ no las tendremos si no se introducen durante el proceso de doblaje) donde sí hay una clara intención humorística (Zabalbeascoa, 20or: 255).

La compensación puede resultar igualmente útil para la traducción de referentes culturales en productos audiovisuales. Según Chaume (2008: 8I), algunos productos como los dibujos animados y las teleseries parecen aceptar más fácilmente esta estrategia para superar posibles restricciones culturales. Este es también el caso de largometrajes como Ali G Indahouse (Ali G anda suelto, 2002) en los que la compensación se emplea con frecuencia para «naturalizar el humor» y compensar pérdidas puntuales o generales de la carga humorística (Botella Tejera, 2006: en línea). En el estudio de productos cinematográficos que recurren con frecuencia a esta naturalización del humor, Botella Tejera (ibídem) llega incluso a hablar de sobretraducción o bipertraducción, un término al que también hacen referencia Díaz Cintas y Remael (2007: 206) al definir la compensación como estrategia de traducción de referentes culturales en subtitulado.

Según afirma Chaume (2008: 80), la compensación también puede resolver los problemas que surjan de la necesidad de emplear un registro concreto en la traducción. Más allá de los problemas específicos que pueda plantear cada texto audiovisual de forma individual, en la mayoría de los casos es preciso que el diálogo suene natural y pueda identificarse con la conversación espontánea. Siguiendo esta reflexión, cabe afirmar que la compensación puede ayudar a mantener la pretendida espontaneidad del diálogo original y conseguir un efecto similar en la audiencia meta. Coincidimos, por tanto, con Díaz Cintas y Remael (2007: 188) en que el traductor puede introducir elementos más 
llamativos y pronunciados en la traducción para compensar la ausencia de intervenciones marcadas en determinados casos. El propósito de los siguientes apartados es ilustrar esta práctica mediante ejemplos extraídos del doblaje de la serie de televisión Friends al español.

\section{EL ESTUDIO DE LA COMPENSACIÓN COMO ESTRATEGIA PARA RECREAR LA CONVERSACIÓN COLOQUIAL ESPONTÁNEA EN COMEDIAS DE SITUACIÓN}

En la traducción para el doblaje de textos audiovisuales de ficción, y en el caso de las telecomedias o comedias de situación en particular, la consecución de unos diálogos creíbles, verosímiles y pretendidamente espontáneos es fundamental y suele ser uno de los parámetros empleados para evaluar la calidad del doblaje de un producto audiovisual (Chaume, 2005: 6). A pesar de la relevancia de este parámetro, varios autores (entre otros, Chaume, 2004; Romero Fresco, 2009; Arampatzis, en este volumen; Baños, 20I2) han corroborado que el traductor no goza de total libertad a la hora de recrear la conversación oral espontánea en doblaje y que, por diversos motivos que no nos detendremos a explicar por cuestiones de espacio, numerosas marcas de oralidad se suelen omitir en los doblajes.

En un estudio previo centrado en comedias de situación (Baños, 2012) se ha corroborado que la omisión de marcas de oralidad suele ser más frecuente en los niveles fonético-prosódico y morfológico, pues las vacilaciones fonéticas, las variedades dialectales y la presencia de rasgos morfológicos no estándar (discordancias, incorrecciones en el uso de tiempos verbales) no suelen tener cabida en el doblaje al español, que se caracteriza por su elevada estandarización lingüística. En el nivel sintáctico, las vacilacio- nes y titubeos, por ejemplo, se suelen verbalizar, explicitar u omitir, y se suele abogar por un orden sintáctico pragmático (ibídem). Aunque la estandarización lingüística también afecta al nivel léxico-semántico, la omisión de marcas de oralidad en este nivel suele ser menor. De hecho, este nivel está «sobrecargado» en comparación con el resto de niveles en los que las concesiones otorgadas al oral espontáneo son escasas. Si se tienen en cuenta estos resultados, es pertinente investigar el papel que desempeña la compensación en la traducción de este rasgo en concreto (la oralidad prefabricada de los textos audiovisuales), según la metodología que se describe a continuación.

\section{Aspectos metodológicos}

Para acometer el estudio del uso de la compensación como estrategia para la consecución de diálogos pretendidamente espontáneos, se ha recurrido al corpus audiovisual compilado en un trabajo anterior (Baños, 20I2). Este corpus está formado por cinco capítulos de la octava temporada de la serie Friends en versión original y los capítulos correspondientes a la versión doblada al español. Se trata, por tanto, de una serie de ficción perteneciente al género de la telecomedia o comedia de situación. La elección de este género en concreto para el estudio de la oralidad prefabricada se debe a que, en estos textos, se intenta imitar o reproducir el español conversacional cotidiano de una manera bastante fiel.

La metodología empleada para la identificación de casos de compensación en el corpus es de corte descriptivo y está centrada en el texto meta y en su posterior comparación con la versión original. El objeto de estudio son las marcas de oralidad que actúan como elemento compensatorio en sí al no estar motivadas por la versión original, y no las marcas de oralidad que 
se pierden o se tienden a omitir en la traducción para el doblaje. Como se ha mencionado anteriormente, este artículo se centra en lo que Harvey (1995: 84) denomina «generalized compensation». En este sentido, consideramos que el uso de determinadas marcas de oralidad no presentes en el texto original se puede entender como parte de una estrategia de compensación global: es decir, ante la «supuesta» imposibilidad o dificultad de reflejar determinados rasgos propios del español coloquial espontáneo en la versión doblada (por ejemplo, vacilaciones fonéticas o uso excesivo de vacilaciones y titubeos), el traductor empleará con frecuencia marcas de oralidad en el nivel léxico-semántico, aunque no estén presentes en la versión original.

Por último, cabe destacar que somos conscientes de la dificultad de encontrar todos los casos posibles de compensación. Por este motivo, el propósito no es realizar un análisis exhaustivo, sino ilustrar algunos de los procedimientos a través de los cuales se materializa la compensación en este tipo de textos y poner de manifiesto su eficacia para conseguir esa pretendida espontaneidad necesaria en doblaje.

\section{RESULTADOS DEL ANÁLISIS DE LA ESTRATEGIA COMPENSATORIA}

\section{Compensación mediante el uso de procedimientos morfológicos de creación léxica en la versión doblada}

Según Gómez Capuz (2000: I43), la sufijación y los procedimientos de acortamiento léxico desempeñan un papel fundamental en la creatividad y expresividad del lenguaje coloquial espontáneo en español. A pesar de ser procedimientos morfológicos, coincidimos con este autor en que estos recursos o marcas de oralidad contribuyen a la creatividad léxica y, por tanto, pueden considerarse recursos propios del nivel léxico-semántico. En el caso de la sufijación, los sufijos diminutivos son muy frecuentes en la conversación coloquial, en la que se suelen emplear con valor de aminoración física, con valor afectivo o emotivo e incluso como atenuantes (ibídem: I45-I46). Aunque los sufijos aumentativos no son tan frecuentes como los diminutivos, también desempeñan un papel importante en la conversación coloquial, sobre todo como intensificadores (ibídem: 146). En el corpus analizado, llama la atención el uso prominente de la sufijación como recurso intensificador y atenuante. El traductor ha decidido aderezar determinados lexemas con sufijos diminutivos (buequecito, poquito, amiguito, un pelín, pequeñita, cabecita, etc.) y, en menor medida (como ocurría en la conversación espontánea), con sufijos superlativos (muchísimo, malisima, tantísimas, buenísimo, etc.) y aumentativos despectivos (narizotas).

Además de observar que la traducción se ajusta a las normas del registro oral del sistema meta, llama la atención que, en un $40 \%$ de los casos analizados, la introducción estas marcas de oralidad no esté motivada directamente por la versión original. $\mathrm{Si}$ bien en algunos casos el traductor ha decidido emplear este tipo de sufijación para traducir intensificadores como so, really y very, que desempeñan un papel fundamental en la serie original (Tagliamonte y Roberts, 2005), en otros su uso se podría entender como parte de una estrategia de compensación global.

Este es el caso del siguiente ejemplo, en el que el adjetivo «bueno» aparece intensificado en la versión doblada mediante procedimientos morfológicos, pero no en la original, en la que la intensificación se realiza exclusivamente en el plano prosódico: 


\section{Ejemplo I}

SERIE: Friends

CAPÍTULO: I92 - El de cuando

Rachel se retrasa TCR: I9.3I

78

Harto de las impertinencias de Rachel, Ross decide acostarse con ella para provocar el parto. Empiezan a besarse $y$ Rachel rompe aguas.

Versión Original (Vo)

Ross: I am good. Okay! Okay! Uh, I got the pillow! I got the bag! You got the keys?

Versión doblada (VD)

Ross: ¡Soy buenísimo! Bien, vamos a ver... Bien, tengo la almohada. Tengo la bolsa. ¿Tienes las llaves?

Algo similar ocurre en el siguiente ejemplo, en este caso de un sufijo diminutivo, cuya introducción — no motivada directamente por el original - aporta espontaneidad a la intervención de Ross (previamente mermada por la omisión de la dubitación «even if-even if») y refuerza el carácter coloquial del producto doblado.

\section{Ejemplo 2}

SERIE: Friends. CAPÍtulo: I9o - El de la fiesta de regalos del bebé. TCR: I6.5I

Ross se enfrenta a la madre de Rachel, que quiere quedarse a vivir con ellos una temporada para cuidar del bebé porque cree que Rachel no está preparada.

$\mathrm{VO}$

Ross: Well uh, y'know what? Even if-even if she doesn't know anything, I do! I have a son. And his mother and I didn't live together, and whenever he was with me I took care of him all the time, by myself.

$\mathrm{VD}$

Ross: Bueno, no importa. Aunque ella no sepa nada, ¡Yo sí! Tengo un hijo. Su madre y yo no vivíamos juntos y siempre que estuvo conmigo cuidé perfectamente de él, yo solito.

Los procedimientos de acortamiento léxico permiten al traductor reforzar el carácter coloquial de las intervenciones de los actores en la versión doblada. A excepción de los hipocorísticos (por ejemplo, Rachel> Rach), se trata de un rasgo no motivado por la versión original en la gran mayoría de los casos. De nuevo, sorprende la elevada frecuencia con la que se recurre a estos procedimientos en la versión doblada, ya que se han identificado 20 ejemplos en todo el corpus. Algunos ejemplos aparecen más de una vez en el corpus. Por ejemplo, movie se suele traducir como "peli» (en lugar de «película») y, relax! como «tranqui». En este sentido, hay que tener en cuenta que cada traductor desarrolla sus propias estrategias de traducción y puede que el traductor de Friends al español haya dado prioridad a estos procedimientos a la hora de compensar la pérdida de otras marcas de oralidad. A continuación se incluye un ejemplo del empleo de estos procedimientos de acortamiento léxico en el corpus, que el traductor parece considerar como «marcas de oralidad coloquial» con las que imprimir naturalidad a los diálogos en español:

\section{EjEMPLO 3}

SERIE: Friends. CAPÍtulo: I9I - El de la clase de cocina. TCR: 14.54

Por casualidad, Joey y Monica acaban en una clase de cocina para principiantes. Al final deciden quedarse para que Monica, que es chef de profesión, pueda destacar y recuperar su autoestima tras recibir una crítica culinaria muy dura.

VO

THE COOKING TEACHER: We're all beginners here. Nobody knows what they're doing.

MoNicA: I do! I'm a professional chef! (The class gasps) Oh relax! It's not a courtroom drama! [...] 
JoEY: Whoa-whoa-whoa-whoa! I don't want to go.

I'm having fun.

THE COOKING TEACHER: Well actually, did either of you pay for this class?

JoEY: Hey-hey-hey, if my friend says it's time to go, it's time to go.

VD

THE COOKING TEACHER: Todos somos principiantes.

Nadie sabe lo que hace.

MonicA: ¡Yo sí! ¡Soy cocinera profesional! (todos se sorprenden) ¡Eh, tranquis!, ¡que no es una tragedia griega! $[\ldots]$

JOEY: ¡Alto, alto, alto! No quiero irme. Esto es diver.

The cooking TeAcher: Por cierto, calguno de los

dos ha pagado la clase?

JOEY: ¡Eh-eh! Si mi amiga dice que es hora de irse, es hora de irse.

\section{Compensación mediante el uso de términos procedentes del argot juvenil en la versión doblada}

Además de mediante neologismos formales como los descritos en el apartado anterior, en algunas ocasiones, el traductor parece compensar la ausencia de marcas de oralidad mediante el empleo de términos y expresiones coloquiales o procedentes del argot juvenil. Este rasgo es característico del léxico coloquial, que presenta una gran capacidad para adoptar voces del argot juvenil (Briz, 1996: 62). En el análisis se han identificado varios casos en los que expresiones de este tipo se utilizan para traducir expresiones de la versión original que son bastante estándar desde el punto de vista léxico.

\section{EJEMPLO 4}

SERIE: Friends. CAPÍtulo: $192-\mathrm{E} 1$ de cuando Rachel se retrasa. TCR: I3.4I

Rachel y Ross cuentan a Phoebe y a Monica que han probado casi todos los remedios alternativos para provocar el parto menos uno: el sexo. Monica ha apostado con Phoebe que la niña nacerá ese día, por lo que anima a Ross y a Rachel a que lo intenten.

VO

MONICA: I'm just saying it's been a really long time for you. I mean, women have needs. Do it, get yours!

Pноеве: Oh I-I don't know about that. No, I think that if the two of you had sex the-the-the repercussions would be catastrophic.

Monica: All right, let's be practical, if Ross isn't willing to do it, he's not the only guy in the world you can have sex with. You can borrow Chandler-Chandler is good!

[...]

Ross: What?! While she's been going through this hell, you've been making money?! You're betting on your friend staying in this misery?!

RACHEL: I'll take that bet.

Ross: What?!

RACHEL: Well, I'm miserable here! I might as well make some money out it!

VD

Monica: Debes tener en cuenta que llevas mucho tiempo sin mojar, en fin, tenemos necesidades. Hazlo, aprovéchate.

Phoebe: Uy, yo no lo tengo tan claro. ¡No! Creo que si hicierais el amor, las...las repercusiones podrían ser catastróficas.

Monica: Vale, seamos prácticos. Si Ross no está dispuesto a hacerlo, no es el único tío del mundo con el que enrollarse. Te presto a Chandler. Es muy bueno en la cama.

[...]

Ross: (A Phoebe) ¿Qué? Mientras ella ha estado aguantando este calvario, ¿tú has estado ganando dinero? ¿Apuestas a que tu amiga seguirá sufriendo?

RACHEL: Acepto la apuesta.

Ross: ¿Qué?

RACHEL: Estoy hecha polvo. Al menos podría ganar un poco de pasta. 
En el ejemplo anterior, el traductor emplea palabras procedentes originariamente del argot juvenil con el fin de emular el registro oral coloquial. Además, se trata de un rasgo que en numerosas ocasiones no está motivado de forma directa por la versión original. En el caso de «pasta», por ejemplo, en la versión original se emplea un término no marcado (money), y lo mismo ocurre con los verbos «mojar» y «enro1larse», traducciones de expresiones bastante estándar desde el punto de vista léxico, como it's been a long time y have sex respectivamente. De nuevo, la inclusión de vocablos de este tipo en los diálogos de la versión doblada de Friends podría formar parte de la estrategia de compensación global, aplicada en el nivel léxico-semántico. Esta tendencia coincide con los resultados obtenidos por Romero Fresco (2006: I47), quien sostiene que el traductor tiende a emplear términos coloquiales a la hora de traducir expresiones o términos no marcados. $\mathrm{El}$ autor recalca que es posible que estos resultados correspondan a preferencias del traductor de Friends, un aspecto en el que merece la pena investigar para comprobar, por ejemplo, si los recursos empleados para compensar la pérdida de oralidad en determinadas intervenciones difieren en función del producto doblado o en función del género. En este caso, los términos argóticos y coloquiales resultan fundamentales para transmitir el tono coloquial del original y crear unos diálogos que suenen cotidianos y familiares.

\section{Compensación mediante el uso de vocativos en la versión doblada}

Al marcar la interacción entre los hablantes, el vocativo desempeña un papel fundamental en la consecución de la oralidad prefabricada del discurso audiovisual, tanto original como doblado. Según el análisis realizado, en la mayoría de las ocasiones los vocativos de la versión doblada se corresponden con vocativos en la versión original. Sin embargo, en diez ocasiones $(6,9 \%$ del total) el traductor ha decidido incluir vocativos que no estaban en la versión en inglés, probablemente para conseguir unos diálogos más naturales y creíbles (en especial mediante el vocativo «tío»), compensar la pérdida de otras marcas de oralidad, así como para satisfacer las necesidades del ajuste. En el siguiente ejemplo, se podría considerar que mediante la inclusión del vocativo se compensa la pérdida de marcas de oralidad tales como las vacilaciones (this is, this is). Como se ha argumentado anteriormente, demostrar de forma sistemática que estamos ante un ejemplo de compensación contigua, desplazada o generalizada en términos de Harvey (1995: 82-83) se torna imposible.

\section{EJEMPLO 5}

SERIE: Friends. CAPÍTUlo: I92 - E1 de cuando Rachel se retrasa. TCR: 18.07

Rachel intenta convencer a Ross para probar lo que les ha recomendado el médico para acelerar el parto: el sexo.

vo

Ross: Okay, enough! This is, this is not going to happen.

VD

Ross: ${ }_{\text {¡No }}$ insistas, Rachel! Te aseguro que eso no va a pasar.

En el siguiente ejemplo, además de compensar la pérdida de marcas de oralidad en otros niveles (la relajación articulatoria de Chandler o la dubitación «huh» en la intervención de Joey), el uso del vocativo «tío», permite encajar la intervención en la boca de Joey y cumplir con 
la isocronía ${ }^{4}$. Asimismo, el vocativo actúa como marcador interpersonal con el que se refuerza la complicidad entre los dos personajes en esta escena.

\section{EJEMPLO 6}

SERIE: Friends. CAPÍTULO: $192-$ El de cuando Rachel se retrasa. TCR: 06.I7

Joey solo tiene una invitación para el estreno de su nueva pelicula y decide invitar a Chandler para agradecerle su apoyo durante su carrera como actor.

$\mathrm{VO}$

CHANDLER: I always knew you were gonna make it. I'm so proud of ya.

JOEY: Thanks. Huh... that means a lot to me.

VD

Chandler: Sabía que ibas a conseguirlo. Estoy orgulloso de ti.

JOEY: Gracias, tío. Significa mucho para mí.

\section{Compensación mediante el uso de unidades fraseológicas en la versión doblada}

La conversación coloquial espontánea se caracteriza por el uso variado de unidades fraseológicas de distinto tipo, sobre todo de locuciones y enunciados fraseológicos (Ruiz Guri1lo, 2000: 169). El análisis del corpus audiovisual ha revelado que estas unidades son también frecuentes en la traducción para el doblaje de comedias de situación. Su inclusión en la versión doblada suele estar motivada por el texto original y ser fruto de la aplicación (con mayor o menor acierto) de determinadas estrategias para la traducción de fraseología. Sin embargo, la inclusión de unidades fraseológicas no está motivada siempre por la versión original y el

4 La isocronía se refiere al ajuste de la traducción a la duración de los enunciados de los personajes de pantalla (Chaume, 2004: 73). traductor parece aprovechar las características del diálogo audiovisual para incluir libremente unidades fraseológicas cuando la situación lo permite. Se ha comprobado que un $46,8 \%$ de las mente motivadas por el texto origen, mientras que en el caso de los enunciados fraseológicos el porcentaje es de un $25,33 \%$. Por tanto, el traductor de Friends tiende a emplear unidades fraseológicas para traducir expresiones no marcadas en el original y, por ejemplo, traduce $I$ don't know como "iyo qué sé!», I know como «iya lo creo!» o Oh, no! como «iqué va!». También encontramos varios casos en el uso de locuciones, pues You'll be perfect for this se traduce como «Te viene como anillo al dedo», I'm totally crazy como «Estoy como una cabra» y he's almost completely deaf como «está sordo como una tapia».

De nuevo, parece que el traductor intenta compensar la pérdida de marcas de oralidad y de coloquialidad en otros niveles de la lengua mediante un uso «excesivo» de unidades fraseológicas y estructuras coloquiales en el nivel léxico. En el siguiente ejemplo, la intervención de Chandler Hardest thing I've ever done in my life, cuya pretendida espontaneidad descansa en el uso de la elipsis y en la relajación articulatoria, se traduce mediante una unidad fraseológica (Casi me meo de la risa), un recurso léxico que resulta idiomático y cómico.

\section{EJEMPLO 7}

SERIE: Friends. CAPÍtulo: I9I - El de la clase de cocina. TCR: 14.54

Phoebe ayuda a Chandler a prepararse para una entrevista de trabajo.

VO

PHoEbE: I see. Nice sidestep on the do do thing by the way.

CHANDLER: Hardest thing I've ever done in my life. 
VD

Pноebe: Entiendo. Por cierto, has esquivado bien lo del meollo.

CHANDler: Casi me meo de la risa.

En algunas ocasiones, las unidades fraseológicas se incluyen en intervenciones que no aparecen en la versión original. En estos casos, el traductor o los participantes en el proceso de doblaje sacan el máximo partido del medio audiovisual, pues aprovechan los cambios de plano o las intervenciones fuera de cámara para conseguir unos diálogos más naturales y espontáneos:

\section{EJEMPLO 8}

SERIE: Friends. CAPÍtulo: I9I, El de la clase de Cocina. TCR: oO.II

Ross llega al Central Perk con un montón de periódicos. Alli están todos menos Monica.

vo

Ross: No, Monica's restaurant got a horrible review in the Post.

ALL: $\underline{\mathrm{Oh} !}$

Ross: I didn't want her to see it, so I ran around the neighborhood and bought all the copies I could find.

[...]

MONICA: Oh my God, this is horrible!

CHANDLER: I'm so sorry, honey.

RACHEL: Yeah but y'know what they say Mon, «There's no such thing as bad press.»

MONICA: You don't think that umm, (reading) "The chef's Mahi Mahi was awful awful," is bad press?

RACHEL: I didn't write it.

Monica: Is he right? Am I really—Am I awful? ALL: No!

VD

Ross: No, en el Post sale una crítica horrible del restaurante de Monica.

RACHEL/PHOEBE: ;Oh no!

JOEY: No me digas!
Ross: Sí, no quiero que la lea, así que he recorrido todo el barrio y he comprado todos los ejemplares. [...]

MONICA: ¡Por Dios, esto es horrible!

CHANDLER: Lo siento, cariño.

RACHEL: Sí, pero ya sabes lo que dicen: que no existe la mala prensa.

Monica: ¿No crees que «El Mahi Mahi de la cocinera estaba fatal, fatal» sea mala prensa?

RACHEL: Yo no lo he escrito.

MONICA: ¿Tiene razón? ¿Tan mal cocino?

Todos: ¡No!

Ross: ¡Qué va!

En el ejemplo anterior, el traductor o los agentes de la cadena del doblaje aprovechan la participación de varios personajes en la intervención, y el hecho de que no todos aparezcan en pantalla, para añadir los enunciados fraseológicos «iNo me digas!» $\mathrm{y}$ «QQué va!», que no aparecen en el original. Este ejemplo, en el que la técnica de traducción de la adición contribuye a la aplicación de una estrategia de compensación general para la consecución de unos diálogos creibles, pone de manifiesto que esta estrategia no sólo es apropiada para compensar la pérdida de un efecto concreto, sino que también permite sacar el máximo partido a las características propias de los textos audiovisuales y a los retos que plantea su traducción.

\section{CONCLUSIONES}

E1 análisis ha puesto de manifiesto que la compensación es una estrategia empleada con frecuencia en el corpus audiovisual objeto de estudio mediante la adición de marcas de oralidad variadas (sobre todo en el plano léxico) que no estaban presentes en el original. Además, se trata de una estrategia útil y apropiada en doblaje ya que, como advierte Chaume (2008: 82), esta se enmarca dentro del método general de la familiarización, que suele ser el método 
adoptado para el doblaje de productos de ficción, donde el propósito es acercar el producto original a los espectadores de la cultura meta. En el estudio de caso presentado en este artículo se ha observado que, mediante técnicas con un efecto compensatorio, el traductor procura seguir las normas que regulan la conversación pretendidamente espontánea o la oralidad prefabricada en el sistema meta. De este modo, se confirma la validez de la compensación como estrategia para recrear la conversación espontánea en doblaje, sobre todo si se tienen en cuenta las convenciones y normas que regulan la traducción de los textos audiovisuales, en los que ciertos recursos o marcas de oralidad no tienen cabida por ser considerados sub-estándar. Dado que dichas convenciones permiten explotar los recursos propios de la conversación coloquial espontánea en el nivel léxico y, en menor medida, en el nivel sintáctico, tiene sentido recurrir a la compensación como estrategia general para conseguir unos diálogos más creíbles y verosímiles. Este razonamiento permite entender el uso «excesivo» de determinadas marcas de oralidad en los textos analizados, así como la afirmación de que el nivel léxico-semántico parece estar «sobrecargado» en comparación con el resto de niveles por lo que respecta al uso de recursos propios del español oral. Por otra parte, los resultados invitan a abordar de distinta forma el estudio del registro del doblaje o dubbese y a centrarnos no solo en lo que se «pierde» en doblaje, sino también en lo que se puede "ganar» si se aprovechan al máximo las «restricciones» o, según se mire, la «flexibilidad» de los textos audiovisuales y de esta modalidad de traducción audiovisual en concreto.

RECIBIDO EN DICIEMBRE DE 2012 ACEPTADO EN ENERO DE 2013 VERSIÓN FINAL DE DICIEMBRE DE 2012

\section{REFERENCIAS BIBLIOGRÁFICAS}

Ávila, A. (1997). El doblaje, Madrid: Cátedra. Baker, M. (2OII) [I992]. In Other Words: A Coursebook on Translation, Londres y Nueva York: Routledge.

Baños, R. (20I2). «La oralidad prefabricada en la traducción para el doblaje y en producciones propias. El caso de Friends y Siete Vidas». En J. J. Martínez Sierra (coord.). Fotografía de la investigación doctoral en traducción audiovisual. Madrid: Bohodón Ediciones, pp. 99-II7.

Baños Piñero, R. \& Chaume, F. (2009), «Prefabricated Orality: A Challenge in Audiovisual Translation» [en línea]. Intralinea, Special Issue: The Translation of Dialects in Multimedia, <http:// www.intralinea.it/specials/dialectrans/ita_more. php?id=76I_o_49_o_M>, [Consulta: 30 sep. 20I2].

Botella Tejera, C. (2006). «La naturalización del humor en la traducción audiovisual (TAV): ¿Traducción o adaptación? El caso de los doblajes de Gomaespuma: Ali G Indahouse» [en línea]. Tonos Digital, I2, <http://www.um.es/tonosdigital/ znumi2/secciones/Estudios\%2oE-Naturalizacion\%2oen\%2oTAV.htm >, [Consulta: 30 sep. $2012]$.

Briz, A. (I996). El español coloquial: situación y uso, Madrid: Arco Libros.

Casetti, F. y di Chio, F. (I99I). Cómo analizar un film (trad. esp. de Carlos Losilla), Barcelona: Paidós.

Chaume, F. (2004). Cine y traducción, Madrid: Cátedra.

Chaume, F. (2005). «Los estándares de calidad y la recepción de la traducción audiovisual». Puentes. Hacia nuevas investigaciones en la mediación intercultural, 6, pp. 5-I2.

Chaume, F. (2008). «La compensación en traducción audiovisual». Quaderns de Filologia. Estudis literaris, I3, pp. 7I-84.

Chesterman, A. (1997). Memes of Translation: the spread of ideas in translation theory, Ámsterdam/ Filadelfia: John Benjamins.

Chiaro, D. (2005). «Verbally Expressed Humour and Translation: An Overview of a Neglected Field». Humor, $18 / 4$, pp. $135^{-}-145$.

Díaz Cintas, J. y A. Remael (2007). Audiovisual Translation: Subtitling, Manchester: St. Jerome.

Gómez Capuz, J. (2000). «La creación léxica II.

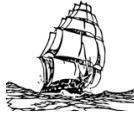




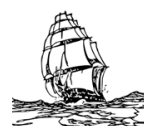

Como se comenta un texto coloquial? Barcelona: Ariel, pp. I43-г68.

Gregory, M. y S. Carroll (1978). Language and Situation. Language Varieties and Their Social Contexts, Londres: Routledge.

Harvey, K. (I995). «A descriptive framework for compensation». The Translator, I/I, pp. 65-86.

Harvey, K. (I998). «Compensation». En M. Baker (ed.). Routledge Encyclopedia of Translation Studies. Londres y Nueva York: Routledge, pp. 37-40.

Hatim, B. y I. Mason (I997). The Translator as Communicator, Londres: Routledge.

Hurtado, A. (200I). Traducción y traductología, Madrid: Cátedra.

Martínez Sierra, J. J. (2008). Humor y traducción. Los Simpson cruzan la frontera, Castellón: Publicacions de la Universitat Jaume I.

Newmark, P. (I99I). About Translation, Clevedon: Multilingual Matters.
Newmark, P. (1988). A Textbook of Translation, London: Prentice Hall.

Romero Fresco, P. (2006). «The Spanish Dubbese: A case of (un)idiomatic Friends». The Journal of Specialised Translation, 6, pp. I34-I5I.

Romero Fresco, P. (2009). "Naturalness in the Spanish Dubbing Language: A case of not-so-close Friends». Meta, 54/1, pp. 49-72.

Ruiz Gurillo, L. (2000). «La fraseología». En A. Briz y Grupo Val.Es.Co (eds.). ¿Cómo se comenta un texto coloquial? Barcelona: Ariel, pp. I69-189.

Salvador, V. (I989). "L'anàlisi del discurs, entre l'oralitat i l'escriptura». Caplletra, 7, pp. 9-31.

Tagliamonte, S. y C. Roberts (2005). «So Weird; So Cool; So Innovative: The Use of Intensifiers in the Television Series Friends». American Speech, 8o/3, pp. 280-30o.

Zabalbeascoa, P. (200I) «La traducción del humor en textos audiovisuales». En M. Duro (coord.), La traducción para el doblaje y la subtitulación. Madrid: Cátedra, pp. 25I-263. 